\title{
Fornix Lesions Decouple the Induction of Hippocampal Arc Transcription from Behavior But Not Plasticity
}

\author{
Bonnie R. Fletcher, ${ }^{1}$ Michael E. Calhoun, ${ }^{2}$ Peter R. Rapp, ${ }^{1}$ and Matthew L. Shapiro ${ }^{1}$ \\ ${ }^{1}$ Fishberg Department of Neuroscience and Alfred B. and Gudrun J. Kastor Neurobiology of Aging Laboratories, Mount Sinai School of Medicine, New York, \\ New York 10029-6574, and 2Department of Cellular Neurology, Hertie-Institute for Clinical Brain Research, University of Tübingen, D-72076 Tübingen, \\ Germany
}

The immediate-early gene (IEG) Arc is transcribed after behavioral and physiological treatments that induce synaptic plasticity and is implicated in memory consolidation. The relative contributions of neuronal activity and learning-related plasticity to the behavioral induction of Arc remain to be defined. To differentiate the contributions of each, we assessed the induction of Arc transcription in rats with fornix lesions that impair hippocampal learning yet leave cortical connectivity and neuronal firing essentially intact. Arc expression was assessed after exploration of novel environments and performance of a novel water maze task during which normal rats learned the spatial location of an escape platform. During the same task, rats with fornix lesions learned to approach a visible platform but did not learn its spatial location. Rats with fornix lesions had normal baseline levels of hippocampal Arc mRNA, but unlike normal rats, expression was not increased in response to water maze training. The integrity of signaling pathways controlling Arc expression was demonstrated by stimulation of the medial perforant path, which induced normal synaptic potentiation and Arc in rats with fornix lesions. Together, the results demonstrate that Arc induction can be decoupled from behavior and is more likely to indicate the engagement of synaptic plasticity mechanisms than synaptic or neuronal activity per se. The results further imply that fornix lesions may impair memory in part by decoupling neuronal activity from signaling pathways required for long-lasting hippocampal synaptic plasticity.

Key words: hippocampus; learning; LTP; Arc; fornix lesion; immediate-early gene

\section{Introduction}

Enduring changes in synaptic efficacy, morphology, and connectivity are widely presumed to be critical for memory consolidation. Stable memory-related synaptic modifications are dependent on both transcription and translation (Flexner et al., 1963; Montarolo et al., 1986; Frey et al., 1988). Protein synthesisindependent transcription of immediate-early genes (IEGs) is a key initial event required for the maintenance of memory and enduring synaptic changes (Abraham et al., 1994; Alberini et al., 1994; Morrow et al., 1999; Guzowski et al., 2000). Expression of the IEG Arc is necessary for certain forms of synaptic plasticity and memory consolidation (Guzowski et al., 2000). Arc transcription is initiated in many behavioral and experimental conditions, including high-frequency stimulation (HFS) (Lyford et al., 1995; Steward et al., 1998), novel environmental exploration (Guzowski et al., 1999; Pinaud et al., 2001; Vazdarjanova and Guzowski, 2004), and learning (Guzowski et al., 2001; Pinaud et al., 2001; Kelly and Deadwyler, 2002, 2003). Arc mRNA transcription and subcellular distribution are tightly regulated: Arc

Received 0ct. 18, 2005; revised Nov. 28, 2005; accepted Dec. 17, 2005.

This work was supported by National Institutes of Health Grants MH65658, DA14166, and AG09973 and the Mount Sinai School of Medicine. We thank Paul Worley and John Guzowski for the generous gift of Arc CDNA Benjamin Schneider, Diana Moga, and Vanja Nadj for technical assistance; and Anna Balk for computer programming.

Correspondence should be addressed to Dr. Matthew L. Shapiro, Mount Sinai School of Medicine, One Gustave Levy Place, Box 1639, New York, NY 10029. E-mail: matthew.shapiro@mssm.edu. DOI:10.1523/JNEUROSCI.4441-05.2006

Copyright $\odot 2006$ Society for Neuroscience $\quad$ 0270-6474/06/261507-09\$15.00/0 appears in the nucleus of hippocampal neurons within 5 min of exploratory behavior, is exclusively in the cytoplasm after $30 \mathrm{~min}$, and is cleared after 60 min (Guzowski et al., 1999). Although Arc is associated with synaptic plasticity and learning, previous studies have not resolved whether behaviorally induced Arc is linked specifically to learning-related plasticity or more broadly to neuronal activity that accompanies behavior. Here, we used fornix lesions to distinguish between these alternatives, testing the prediction that a manipulation known to selectively impair hippocampal learning should also block hippocampal Arc transcription.

The fornix provides bidirectional connectivity between the hippocampus and subcortical structures (Swanson and Cowan, 1977; Cassel et al., 1997). Fornix lesions reduce long-term potentiation (LTP) persistence in the dentate gyrus (Buzsaki and Gage, 1989; Nakao et al., 2001), impair hippocampus-dependent spatial learning, but spare hippocampus-independent cue-approach learning (Nilsson et al., 1987; Packard et al., 1989; Sutherland and Rodriguez, 1989; Sziklas and Petrides, 2002; Gaffan et al., 2003). The behavioral correlates of hippocampal neuronal activity are essentially maintained after fornix lesions, such that pyramidal cells reveal place fields (Miller and Best, 1980; Shapiro et al., 1989). Thus, fornix lesions provide a model in which hippocampal plasticity and learning are compromised even as the neuronal firing patterns organized by behavior persist.

Experience-dependent induction of Arc expression was disrupted by unilateral lesions of the fornix (Temple et al., 2003). To 
clearly define the requirements for behavioral engagement of Arc transcription, we compared Arc mRNA expression in normal rats with those given bilateral fornix lesions. Neither performance of a water maze task that could be solved by either hippocampus-dependent or -independent strategies nor exploration of novel environments induced hippocampal Arc in animals with fornix lesions; in contrast, medial perforant path (MPP) potentiation induced Arc normally. The overall pattern suggests that changes in Arc expression in the hippocampus are not correlated with changes in cellular activity that accompany behavior per se, or learning supported by other brain systems, but are specifically correlated with learningrelated plasticity that requires the hippocampus.

\section{Materials and Methods \\ Animals}

Male Long-Evans rats (350-400 g; Harlan, Indianapolis, IN) were housed individually and maintained on a $12 \mathrm{~h} \mathrm{light/dark} \mathrm{cycle} \mathrm{with} \mathrm{food}$ and water available ad libitum. Animals were allowed at least 1 week to acclimate to the vivarium before surgery. All procedures with animals met National Institutes of Health guidelines and were approved by the Mount Sinai Institutional Animal Care and Use Committee.

\section{Experimental design}

All rats were given surgery, tested in the water maze, and assessed histologically for lesion completeness and Arc induction. The treatments, order, and number of rats are illustrated in Table 1.

\section{Surgery}

Rats were anesthetized with $2.5-5.0 \%$ isoflurane (Forane; Baxter, Deerfield, IL) and placed in a stereotaxic apparatus with the incisor bar at 5.0 $\mathrm{mm}$ below the interaural line. The scalp was cut and retracted, and holes were drilled in the skull at anteroposterior (AP) $-1.5 \mathrm{~mm}$ and mediolateral (ML) \pm 0.8 and $\pm 2.2 \mathrm{~mm}$ relative to bregma (Paxinos and Watson, 1986). The dura was pierced, and two $0.254-\mu \mathrm{m}$-diameter nickelchromium-plated nichrome electrodes (Leico Industries, New York, $\mathrm{NY}$ ) with $\sim 0.5 \mathrm{~mm}$ of insulation removed from the electrode tip were lowered $4.8 \mathrm{~mm}$ from the skull surface. Electrodes were lowered into the most medial holes and the lateral holes. Lesions were made by passing radiofrequency current between the medial electrodes $(10 \mathrm{~mA}, 40 \mathrm{~s})$ and between the lateral electrodes $(8 \mathrm{~mA}, 40 \mathrm{~s})$. In the sham surgery condition, electrodes were lowered, but no current was passed. The scalp was then closed with staples, and animals were returned to the colony for a recovery period of $14 \mathrm{~d}$.

\section{Morris water maze}

The water maze was a circular tank $(170 \mathrm{~cm}$ diameter, $63.5 \mathrm{~cm}$ height $)$ filled $38 \mathrm{~cm}$ deep with water $\left(23 \pm 1^{\circ} \mathrm{C}\right)$. White tempera paint (Dick Blick, Galesburg, IL) was added to the water to make it opaque. A clear Plexiglas platform $(10.5 \times 10.5 \mathrm{~cm})$ was submerged $2 \mathrm{~cm}$ below the surface of the water for hidden and cued trials. For cued trials, the platform location was signaled by a black cue on top of the platform and by another black cue suspended $35 \mathrm{~cm}$ above the platform. These cues were removed during hidden trials. The platform location was constant throughout testing, except for the probe trial. A custom-made computerized tracking system recorded and analyzed the swim paths.

All rats were tested on a novel $1 \mathrm{~d}$ water maze task to assess hippocampal-dependent and -independent learning (sham, $n=17$; lesion, $n=19$ ) and to induce Arc transcription in a subset of animals. Rats were trained initially to swim to the visibly cued platform to escape the water. The platform location remained constant across trials; thus, rats could escape efficiently by learning to approach the visible cue, the spatial location of the platform, or both. After the initial training phase, the visible cue was removed from the platform on alternate trials, forcing the animals to learn the spatial location of the platform to escape efficiently. To determine which strategy the rats used to escape, a probe trial was given $30 \mathrm{~min}$ after the last cued trial. Spatial bias was measured as the amount of time rats spent in the goal quadrant compared with other quadrants during the probe trial. This parameter is influenced by decreased swim speed in the vicinity of the former platform location, yielding a more sensitive measure of acquired spatial bias than path length per quadrant. Rats that learn the location of the platform show a bias for the quadrant of the maze in which the platform was located, whereas rats that only learn to approach the visible cue show no quadrant preference.

The training session consisted of 15 trials with a $15 \mathrm{~s}$ intertrial interval (ITI) and a probe trial 30 min later. Trials $1-9,11,13$, and 15 were cued, and the platform was hidden on trials 10,12, and 14. Rats were allowed 60 s to locate the platform on trials 1-15; animals that failed to escape in the allotted time were guided to the platform by the experimenter. Rats remained on the platform for $15 \mathrm{~s}$ and were returned to a holding cage between trials and for the $30 \mathrm{~min}$ ITI preceding the probe trial. During the probe trial, the platform was removed from the maze and rats were allowed to swim for $60 \mathrm{~s}$. Training performance was measured by path length (the total distance swum from start location to platform). Animals used to assess Arc induction resulting from water maze training were killed immediately after completion of the probe trial (sham, $n=4$; lesion, $n=4$ ). All other animals were returned to the colony after water maze training. Animals designated as home-cage controls (sham, $n=2$; lesion, $n=2$ ) were killed $24 \mathrm{~h}$ after completion of the probe trial.

\section{Novel environment exposure}

One day after water maze testing, rats in the novel environment exposure group (sham, $n=8$; lesion, $n=8$ ) explored two novel environments, following a protocol known to produce Arc expression in both the nucleus and the cytoplasm of hippocampal neurons (Guzowski et al., 1999). Before exposure to novel environments, the animals were habituated to the testing room for $6+\mathrm{h}$. During this time, they remained in their home cages and were maintained on the same light/dark cycle as that in the home colony. Environment A was a white $76 \times 76 \mathrm{~cm}$ square with 30.5 $\mathrm{cm}$-high walls. Environment B was a circular area, $76 \mathrm{~cm}$ in diameter, with 30.5-cm-high black walls and a white floor. Constellations of contrasting black or white geometric shapes were attached to the walls of each environment, and the chambers were cleaned thoroughly between exposures. Environmental exposure consisted of $5 \mathrm{~min}$ in environment A, 25 min back in the home cage, followed by 5 min in environment A or B. Immediately after the second environmental exposure, animals were killed.

\section{MPP stimulation}

Rats (sham, $n=3$; lesion, $n=5$ ) were anesthetized with $1.5 \mathrm{~g} / \mathrm{kg}$ urethane (Sigma, St. Louis, MO) and placed in a stereotaxic frame. Anesthesia was supplemented as needed with isoflurane. The scalp was cut and retracted, and the skull and dura were removed dorsal to the hippocampus and entorhinal cortex. A bipolar, concentric, stainless steel recording electrode (Frederick Haer, Bowdoinham, ME) was lowered into the hilus of the dentate gyrus at AP $-3.6 \mathrm{~mm}$ and ML $2.2 \mathrm{~mm}$. An epoxylitecoated insect pin stimulating electrode was lowered into the MPP at approximately AP $-8.1 \mathrm{~mm}$ and ML $4.4 \mathrm{~mm}$. Both electrodes were positioned dorsoventrally to elicit a maximal population spike (PS). An analog stimulator (A-M Systems, Bellevue, WA) was used to generate 
biphasic stimulation pulses. Responses were filtered $(1 \mathrm{~Hz}$ to $1 \mathrm{kHz})$ and amplified $(1000 \times)$ by a differential AC amplifier (A-M Systems). Experimenter's Workbench software (BrainWave Systems, Thornton, CO) was used to collect and analyze all responses. Input/output (I/O) curve and baseline recordings ( $50 \%$ of the maximal PS on the I/O curve) were taken before and after HFS (eight pulses at $400 \mathrm{~Hz}$ every $10 \mathrm{~s}$ for $2 \mathrm{~h} ; 50 \%$ maximal PS). Animals were killed immediately after completion of the last baseline recording.

\section{Tissue preparation}

Animals were deeply anesthetized with 5\% isoflurane and perfused transcardially with $\sim 70 \mathrm{ml}$ of cold $0.1 \mathrm{M}$ PBS, followed by $450 \mathrm{ml}$ of cold $4 \%$ paraformaldehyde in $0.1 \mathrm{M} \mathrm{PBS}, \mathrm{pH} 7.2-7.4$, at a rate of $35 \mathrm{ml} / \mathrm{min}$. Brains were removed and postfixed in perfusate at $4^{\circ} \mathrm{C}$ overnight. This was followed successively by cryoprotection in 10 and $20 \%$ glycerol in $0.1 \mathrm{M}$ phosphate buffer $(\mathrm{PB})$ at $4^{\circ} \mathrm{C}$ overnight. Brains were blocked and frozen for $20 \mathrm{~min}$ in a 2-methylbutane (Fisher Scientific, Pittsburgh, PA) bath surrounded by dry ice. Tissue was stored at $-80^{\circ} \mathrm{C}$ until sectioning on a freezing/sliding microtome. Fifty-micrometer sections were taken from the anterior limit of the fornix through the rostrocaudal extent of the hippocampus. Sections were stored in RNase-free PBS at $4^{\circ} \mathrm{C}$ or cryoprotectant solution ( $30 \%$ glycerol and $30 \%$ ethylene glycol in $0.1 \mathrm{M} \mathrm{PB}$ ) at $-80^{\circ} \mathrm{C}$ until Arc in situ hybridization, acetylcholinesterase (AchE) histochemistry, vesicular acetylcholine transporter (VAchT) immunohistochemistry, and Nissl staining. The latter markers were used to document the extent and selectivity of the fornix lesions in experimental animals.

\section{Nonradioactive in situ hybridization}

Reagents were obtained from Sigma unless noted otherwise. Solutions were filtered, treated with $0.1 \%$ diethyl pyrocarbonate, and autoclaved before use, except solutions containing proteins or EDTA, which were prepared using RNase-free materials and dissolved in RNase-free water. Fixation and pretreatment of tissue. Sections were mounted onto slides (Superfrost Plus; Fisher Scientific), allowed to dry, and postfixed for 20 min in $4 \%$ paraformaldehyde in PBS at $4^{\circ} \mathrm{C}$, followed by two washes in PBS. Sections were permeabilized with $10 \mathrm{mg} / \mathrm{ml}$ proteinase $\mathrm{K}$ in TE [10 mM Tris-HCl (Fisher Scientific) and 1 mm EDTA, pH 7.5] and washed briefly in PBS. Tissue was then equilibrated in $0.1 \mathrm{M}$ triethanolamine (Fluka, Buchs, Switzerland) and acetylated with $0.25 \%$ acetic anhydride (Fluka) in $0.1 \mathrm{~m}$ triethanolamine. After two washes in $2 \times$ SSC [ $1 \times: 3 \mathrm{M}$ $\mathrm{NaCl}$ and $0.3 \mathrm{M}$ sodium citrate (both from Fisher Scientific), $\mathrm{pH}$ adjusted to 7.0 with citric acid], sections were dehydrated through graded alcohols and defatted in ethanol:chloroform (Fisher Scientific). Tissue was rehydrated through graded alcohols and two washes in $2 \times$ SSC. Sections were then outlined with a DPX (BDH Laboratory Supplies, Poole, UK) guard wall before hybridization.

RNA probes. Digoxygenin-labeled sense and antisense RNA probes were generated from nearly full-length Arc cDNA (Lyford et al., 1995) inserted into a pBluescript II SK plasmid (Stratagene, La Jolla, CA). Sense and antisense probes were transcribed using T3 and T7 RNA polymerase (Roche, Indianapolis, IN), respectively. Probe concentration was estimated by spotting serial dilutions of probe and standard RNA on membranes, immunodetection with anti-DIG Fab fragments conjugated to alkaline phosphatase (Roche), and colorization with substrate solution [337 $\mu \mathrm{g} / \mathrm{ml}$ nitroblue tetrazolium (Roche), $175 \mu \mathrm{g} / \mathrm{ml}$ 5-bromo-4chloro-3-indolyl phosphate (Roche) in $100 \mathrm{~mm} \mathrm{NaCl}, 100 \mathrm{~mm}$ Tris-HCl, and $50 \mathrm{~mm} \mathrm{MgCl}_{2}$ (Fisher Scientific), $\mathrm{pH}$ 9.5].

Hybridization. Probe RNA was diluted to $100 \mathrm{ng} / \mathrm{ml}$ in hybridization buffer [ $50 \%$ deionized formamide, $2 \times$ SSC, $1 \times$ Denhardt's solution $(1 \%$ bovine serum albumin [BSA], $1 \%$ polyvinylpyrolidone, and $1 \%$ Ficoll), $10 \%$ dextran sulfate, $0.5 \mathrm{mg} / \mathrm{ml}$ yeast tRNA, and $0.5 \mathrm{mg} / \mathrm{ml}$ salmon sperm DNA] and denatured at $80^{\circ} \mathrm{C}$ for 5 min before application to tissue. Sections were incubated $16-20 \mathrm{~h}$ at $59^{\circ} \mathrm{C}$ in a humid chamber.

Posthybridization/stringency washes. Excess hybridization buffer was drained, and slides were washed twice in $2 \times$ SSC plus $1 \mathrm{~mm}$ EDTA. Sections were then treated with $10 \mu \mathrm{g} / \mathrm{ml}$ RNase A in TE plus $0.5 \mathrm{M} \mathrm{NaCl}$ and washed twice with $2 \times$ SSC plus 1 mM EDTA. Tissue was then subjected to stringency washes in $0.1 \times$ SSC plus $1 \mathrm{~mm} \mathrm{EDTA}$ at $59^{\circ} \mathrm{C}$ and $0.5 \times$ SSC at room temperature. After PBS washes, the tissue was blocked with $10 \%$ BSA in PBS.
Immunodetection. Sections were incubated with anti-DIG Fab fragments conjugated to alkaline phosphatase (Roche) diluted 1:1000 in 10\% BSA in PBS. After washes with PBS, TBS (in mM: 84 Tris- $\mathrm{HCl}, 16$ Trisbase, and $154 \mathrm{NaCl}$ ), and equilibration in detection buffer (in mм: 100 $\mathrm{NaCl}, 100$ Tris- $\mathrm{HCl}$, and $50 \mathrm{MgCl}_{2}, \mathrm{pH}$ 9.5), probe labeling was detected by incubation in substrate solution. Developed sections were washed with TE and allowed to dry before coverslipping with VectaShield (Vector Laboratories, Burlingame, CA).

\section{Radioactive in situ hybridization}

All solutions were prepared as described for nonradioactive in situ hybridization.

Tissue pretreatment. Sections were mounted onto slides, allowed to dry, and washed in $0.1 \mathrm{~m}$ glycine in $0.1 \mathrm{M} \mathrm{PB}$, followed by a wash in $0.1 \mathrm{M}$ $\mathrm{PB}$. Tissue was then permeablized with $1 \mu \mathrm{g} / \mathrm{ml}$ proteinase $\mathrm{K}$ in $\mathrm{TE}$, followed by acetylation in $0.25 \%$ acetic anhydride in $0.1 \mathrm{M}$ triethanolamine. Tissue was then washed in $2 \times$ SSC before hybridization.

RNA probes. ${ }^{35} \mathrm{~S}$ (PerkinElmer, Sheldon, CT)-labeled sense and antisense RNA probes were generated using T3 and T7 RNA polymerases, respectively, from nearly full-length Arc cDNA (Lyford et al., 1995) inserted into a pBluescript II SK plasmid (Stratagene). Probe concentration was measured by scintillation.

Hybridization. Probe RNA was diluted to 10,000 counts per milliliter of hybridization buffer plus $20 \mathrm{~mm}$ DTT (Roche) and applied to the tissue. Sections were incubated $16-20 \mathrm{~h}$ at $59^{\circ} \mathrm{C}$ in a humid chamber.

Posthybridization/stringency washes. Excess hybridization buffer was drained, and slides were washed twice in $4 \times$ SSC plus $5 \mathrm{~mm} \mathrm{DTT} \mathrm{at} 59^{\circ} \mathrm{C}$. Sections were then treated with $20 \mu \mathrm{g} / \mathrm{ml}$ RNase A in TE plus $0.5 \mathrm{M} \mathrm{NaCl}$ and washed four times with $2 \times$ SSC plus 5 mM DTT. Tissue was then subjected to two stringency washes in $0.5 \times$ SSC plus $5 \mathrm{~mm} \mathrm{DTT}$ at $59^{\circ} \mathrm{C}$ and one wash in $0.1 \times$ SSC plus $5 \mathrm{~mm}$ DTT at room temperature. Tissue was allowed to dry before exposure to Eastman Kodak BioMax MR film (Amersham Biosciences, Piscataway, NJ) for $\sim 18 \mathrm{~h}$.

Quantification. Films were developed with Eastman Kodak GBX Developer, fixed in Eastman Kodak Professional Fixer, rinsed in tap water, and dried before scanning at 1200 pixels per inch. Image J software (http://rsb.info.nih.gov/ij/) was used to trace and quantify the mean optical density of the regions of interest from four sections $600 \mu \mathrm{m}$ apart, starting at the dorsal pole of the hippocampus. Optical densities in hippocampal areas CA1 and CA3 were assessed in tissue from the water maze induction group. Optical densities in the granular cell layers and molecular layers of the dentate gyrus were assessed in tissue from the MPP induction group. Optical densities collected from several different films, as with the water maze induction group, were normalized by calibration to radioactive standards (Amersham Biosciences). Optical densities collected from the same film, as with the MPP potentiation group, were not calibrated.

\section{AChE histochemistry}

To assess the extent of hippocampal denervation after fornix lesion, AchE-positive fibers were visualized according to standard procedures (Hedreen et al., 1985). Free-floating sections were then mounted on slides (Superfrost Plus; Fisher Scientific), allowed to dry, dehydrated through a graded series of alcohols, cleared in xylenes, and coverslipped with DPX.

\section{VAchT immunohistochemistry and gold-silver toning}

To further assess hippocampal cholinergic denervation caused by fornix transection, the tissue was probed for VAchT immunoreactivity. Freefloating sections were washed with $0.01 \mathrm{M}$ PBS and blocked with $5 \%$ horse serum, $1 \%$ BSA, and $0.1 \%$ Triton X-100 (Fisher Scientific) in $0.01 \mathrm{~m}$ PBS. Sections were incubated with anti-VAchT primary antibody (ImmunoStar, Hudson, WI), diluted 1:50,000 in 3\% horse serum, 1\% BSA, and $0.1 \%$ Triton X-100 in $0.01 \mathrm{M} \mathrm{PBS}$ at $4^{\circ} \mathrm{C}$ for $48 \mathrm{~h}$. After several washes with 0.01 м PBS, sections were incubated with biotinylated horse anti-goat secondary antibody (Vector Laboratories) diluted 1:500 in 3\% horse serum and $1 \% \mathrm{BSA}$ in $0.01 \mathrm{M} \mathrm{PBS}$, for $2 \mathrm{~h}$ at $4^{\circ} \mathrm{C}$. Sections were washed with $0.01 \mathrm{M}$ PBS and incubated with avidin-peroxidase complex using the Biostain Super ABC kit (Biomeda, Foster City, CA) for $2 \mathrm{~h}$ at room temperature. After washes with $0.01 \mathrm{M}$ PBS, sections were developed with 

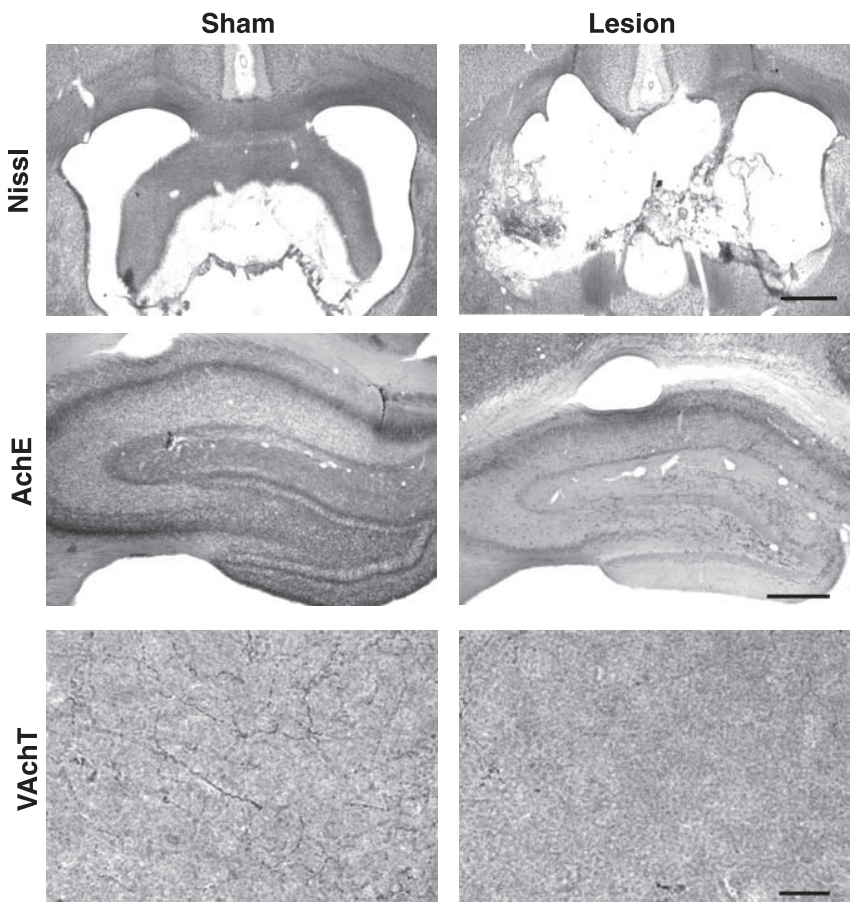

Figure 1. Histological verification of fornix lesions. Nissl staining of the fornix in representative sham and lesion animals is shown. AchE immunohistochemistry reveals the extent of hippocampal cholinergic innervation. The normal innervation pattern of cholinergic fibers is shown in tissue from a sham animal. The hippocampus of a lesioned animal shows almost no cholinergic innervation. VAchT immunoreactivity in the stratum radiatum confirms the near complete loss of cholinergic inputs to the hippocampus as a result of a fornix lesion. Scale bars: top and middle rows, $1 \mathrm{~mm}$; bottom row, $10 \mu \mathrm{m}$.

$1 \mathrm{mg} / \mathrm{ml}$ diaminobenzidine and $0.01 \%$ hydrogen peroxide in $0.01 \mathrm{M}$ PBS. After several washes, sections were mounted and dried overnight.

Specific immunoreactivity was enhanced by gold-silver toning resulting in optimal signal-to-noise visualization of VachT-positive fibers in the hippocampus. First, sections were defatted in ethanol:chloroform, followed by rehydration through graded ethanol washes. Slides were then incubated in $1 \%$ silver nitrate at $56^{\circ} \mathrm{C}$ for $45 \mathrm{~min}$ before neutralization with $0.6 \%$ ammonium hydroxide. After a brief water wash, slides were incubated in $0.2 \%$ gold chloride for $10 \mathrm{~min}$ and rinsed again in water before incubation with $5 \%$ sodium thiosulfate (Fisher Scientific) for 10 min. Slides were then dehydrated and coverslipped.

\section{Lesion verification}

The extent and selectivity of fornix lesions were examined using Nissl staining, AchE histochemistry, and VAchT immunoreactivity (Fig. 1). The size and completeness of the lesions were evaluated by microscopic inspection of Nissl-stained tissue. The completeness of the fornix transections was further assessed by the extent of cholinergic denervation as indicated by AchE-labeled fibers in the hippocampus. Cholinergic denervation was also confirmed using VAchT immunostaining, a more selective marker of cholinergic fibers.

\section{Results}

Fornix lesions impaired spatial but not cue approach learning Rats given sham surgery had an intact fornix and were designated "sham" animals $(n=17)$. Only rats with complete hippocampal cholinergic denervation and bilateral transection of the fornix in Nissl-stained material were included in the lesion group $(n=19)$.

Both lesion and sham groups acquired the cued task at the same rate (Fig. 2A) (repeated-measures ANOVA; main effect of trials 1-9: $F_{(8,272)}=27.196, p<0.0001$; main effect of lesion: $F_{(1,34)}=1.709, p=0.20$; interaction effect of lesion by trials: $\left.F_{(8,272)}=0.536, p=0.83\right)$ and maintained similar levels of as-
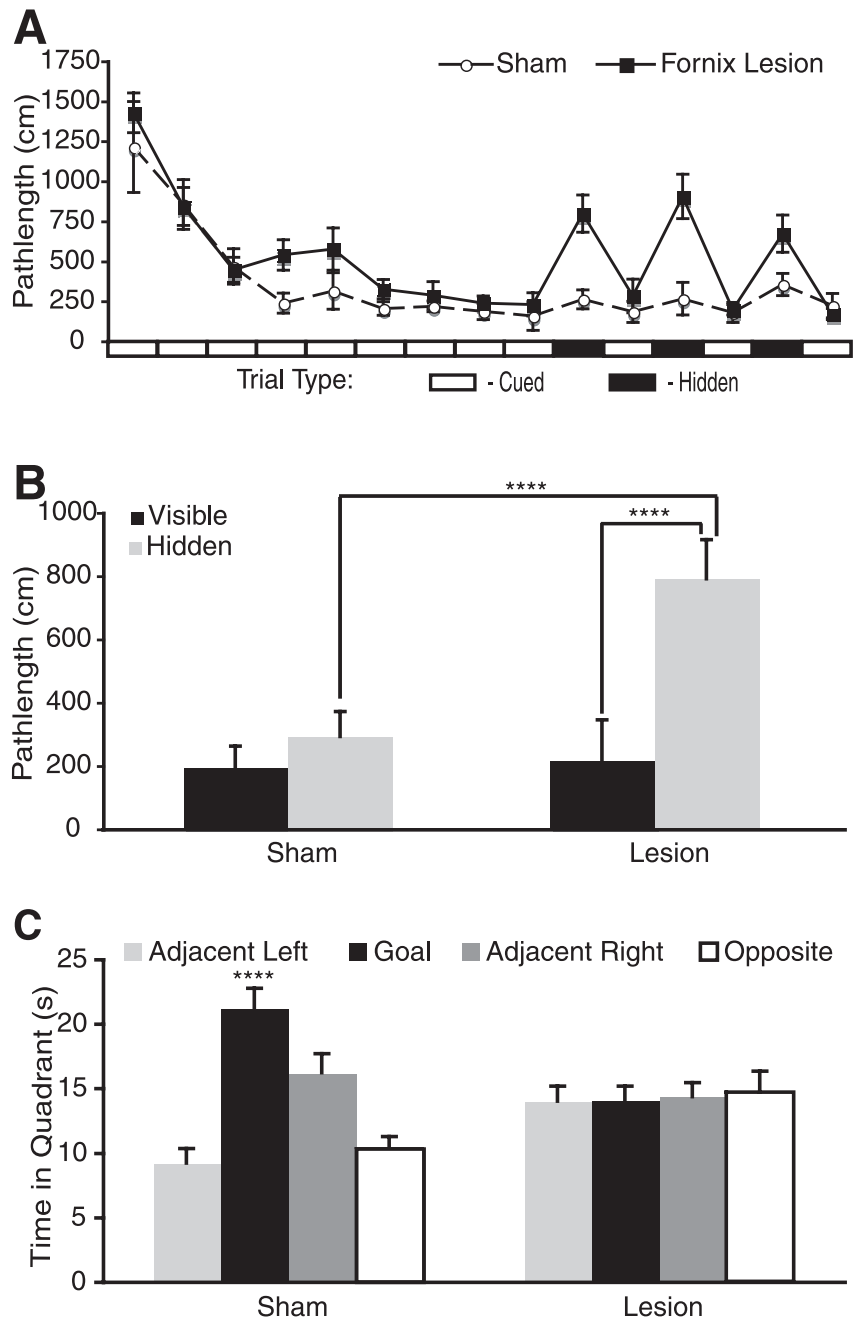

D
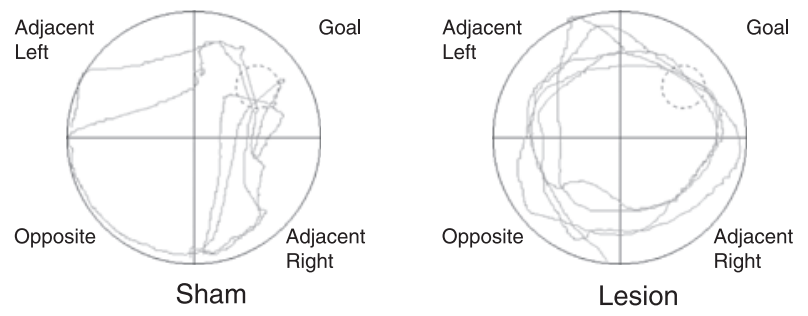

Figure 2. Fornix lesions impair spatial learning but not cue learning. $\boldsymbol{A}$, Sham $(\bigcirc)$ and lesion (ם) groups performed similarly on cued trials (open rectangles, ordinate axis). Performance of the lesion group was impaired on hidden platform trials (filled rectangles, ordinate axis). $\boldsymbol{B}$, The mean performance of the lesion group was significantly worse on the three hidden platform trials than on the last three visible trials (Scheffe's test; $p<0.0001$ ) and significantly worse than the sham group (Scheffe's test; $p<0.0001$ ). C, During the probe trial, sham animals showed a significant bias for the goal quadrant. Lesion animals showed no quadrant bias. $\boldsymbol{D}$, Representative probe trial swim paths from sham and lesion animals. ${ }^{* * *} p<0.0001$, statistically significant. Error bars indicate SEM.

ymptotic performance on the cued trials across the interleaved spatial trials (repeated-measures ANOVA for cued trials 11, 13, and 15; main effect of lesion: $F_{(1,34)}=0.037, p=0.85$; main effect of trial: $F_{(2,68)}=0.444, p=0.64$; interaction effect of trial by lesion: $F_{(2,68)}=1.239, p=0.30$ ) (Fig. $2 A$ ). In contrast, rats with fornix lesions performed significantly worse than shams in the spatial task (repeated-measures ANOVA of hidden platform trials 10, 12, and 14; main effect of lesion: $F_{(1,34)}=28.006, p<$ 
0.0001; main effect of trial: $F_{(2,68)}=0.168, p=0.85$; interaction effect of trial by lesion: $F_{(2,68)}=1.407, p=0.25$ ) (Fig. $2 B$ ). Thus, lesions of the fornix impaired spatial but not cue learning.

\section{Fornix lesions impair spatial memory}

Probe trial quadrant preference differed significantly between groups (repeated-measures ANOVA; main effect of quadrant: $F_{(3,102)}=6.197, p<0.001$; interaction effect of quadrant by lesion: $F_{(3,102)}=6.596, p=0.001$ ) (Fig. $2 C$ ). The sham group spent significantly more time in the goal quadrant compared with the opposite (Scheffe's test; $p<0.0001$ ) and adjacent (Scheffe's test; $p<0.0001$ ) left quadrants. Lesion animals did not show a significant quadrant preference. These data confirm and extend previous studies demonstrating impaired spatial learning and intact cue learning after fornix lesions (Olton et al., 1987; Packard et al., 1989; Sutherland and Rodriguez, 1989; McDonald and White, 1993). Sham animals performed similarly on the three hidden trials and the last three cued trials (ANOVA; main effect of trial type: $\left.F_{(1,100)}=2.308, p=0.1319\right)$ (Fig. $2 B$ ), indicating that they had learned the spatial location of the platform during previous cued trials. Animals with fornix lesions performed significantly worse on the three hidden trials than the last three cued trials (ANOVA; main effect of trial type: $F_{(1,112)}=50.093, p<0.0001$ ) (Fig. $2 B$ ), indicating that they had not learned the spatial location of the platform during previous cued trials. Together, the performance on the hidden and probe trials demonstrated that under conditions in which performance is similar (i.e., cued trials), sham and lesion animals learned different information.

\section{Fornix lesions prevent induction of hippocampal Arc} expression in response to exploration of a novel environment One day after behavioral testing in the water maze, the rats assigned to the environmental exploration group were allowed to explore two novel environments for $5 \mathrm{~min}$ each, separated by a period of $25 \mathrm{~min}$ when the animals were returned to their home cages. This procedure induces Arc mRNA in the hippocampus of normal animals (Guzowski et al., 1999; Vazdarjanova and Guzowski, 2004) but not in the hippocampus ipsilateral to a unilateral fornix lesion (Temple et al., 2003).

Rats given sham lesions showed both nuclear and cytoplasmic Arc RNA expression in all hippocampal cell fields after novel environmental exposure (Fig. 3), similar to previous descriptions (Guzowski et al., 1999; Vazdarjanova and Guzowski, 2004). In contrast, rats with fornix lesions had minimal Arc expression in the hippocampus. Sense probes produced no labeling (data not shown). Thus, fornix lesions blocked experience-dependent induction of Arc expression in the hippocampus. These data are consistent with previous work demonstrating a disruption of experience-dependent induction of Arc expression after unilateral fornix lesions (Temple et al., 2003).

\section{Fornix lesions prevent induction of hippocampal Arc expression in response to water maze training}

To extend previous findings and determine whether a task that engages the hippocampus of normal animals results in Arc expression in rats with fornix lesions, we examined Arc expression after water maze training. Rats given sham lesions showed both nuclear and cytoplasmic Arc mRNA expression in all hippocampal cell fields as a result of water maze training (data not shown). In contrast, hippocampal Arc expression in rats with complete fornix lesions and home-cage control animals was minimal.

Arc mRNA transcription in hippocampal subfields CA1 and CA3 was quantified by in situ autoradiography (Fig. $4 B$ ). Sham
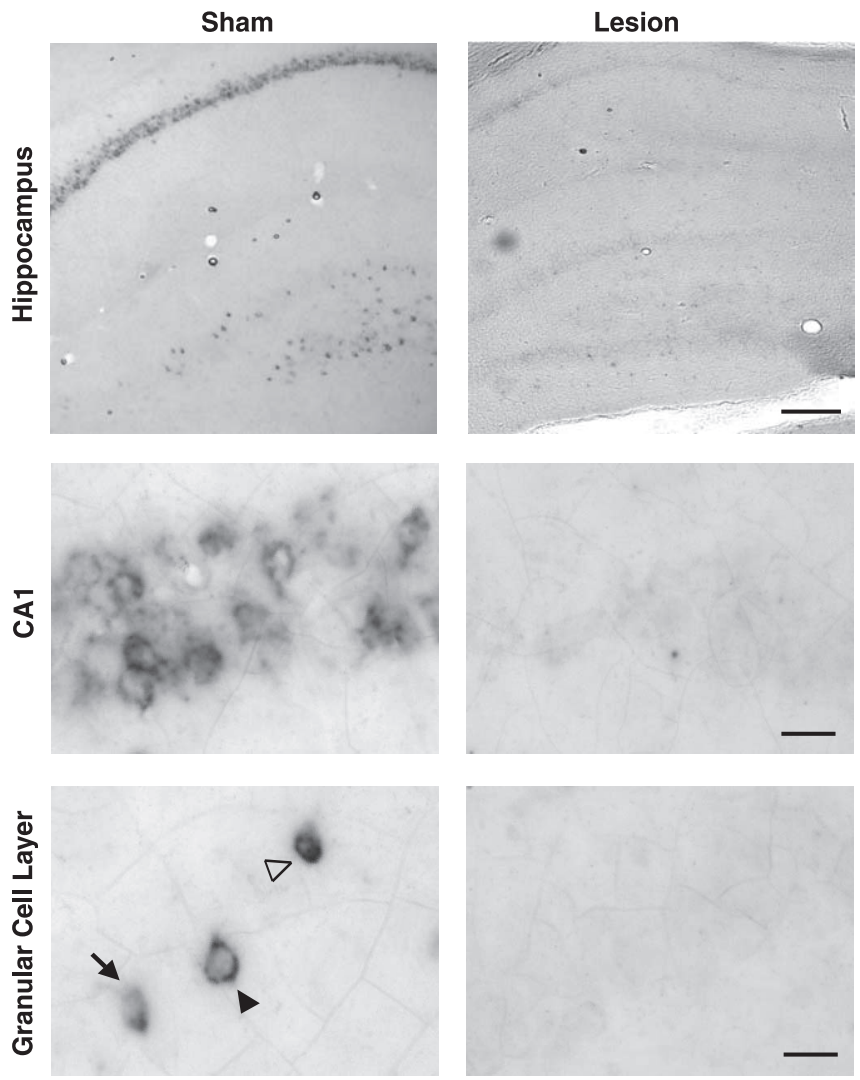

Figure 3. Fornix lesions prevent induction of hippocampal Arc mRNA after environmental exploration. Arc mRNA expression was evident in the CA1 and dentate gyrus of sham animals but not in rats with complete fornix lesions. Compartmental Arc expression in the dentate granule cells can be seen in the nucleus (arrow), cytoplasm (filled arrowhead), and both the nucleus and the cytoplasm (open arrowhead). Scale bars: top row, $1 \mathrm{~mm}$; middle and bottom rows, $10 \mu \mathrm{m}$.

and lesion home-cage controls were collapsed because expression did not differ in these groups across either CA1 or CA3 (Scheffe's test: CA1, $p=0.56$; CA3, $p=0.94)$. After water maze training, however, Arc expression levels varied significantly between groups in CA1 (main effect of group: $F_{(2,9)}=14.274, p=0.0016$ ) (Fig. $4 A$ ). In the sham group, Arc expression was significantly higher than in both the lesion group (Scheffe's test; $p=0.0045$ ) and home-cage controls (Scheffe's test; $p=0.0039$ ). Arc expression levels in CA1 did not differ between the water maze-trained fornix lesion group and home-cage controls (Scheffe's test; $p=$ 0.99). Despite the numerical trend toward higher values in the sham group, Arc expression levels in CA3 did not vary significantly between groups (main effect of group: $F_{(2,9)}=2.672, p=$ 0.12 ) (Fig. 4A). These data indicate that a task that engages the hippocampus of normal animals did not induce Arc expression in the hippocampus of animals with fornix lesions.

\section{Fornix lesions do not prevent induction of Arc expression in response to MPP potentiation}

Fornix lesions could prevent the behavioral induction of Arc either by decoupling behavior from crucial intracellular signaling mechanisms or by producing damage that renders the hippocampus incapable of upregulating Arc transcription. To determine whether the hippocampus could induce Arc transcription after fornix lesions, we delivered HFS to the perforant path using a protocol known to induce LTP and Arc transcription in intact animals (Steward et al., 1998; Steward and Worley, 2001). HFS 


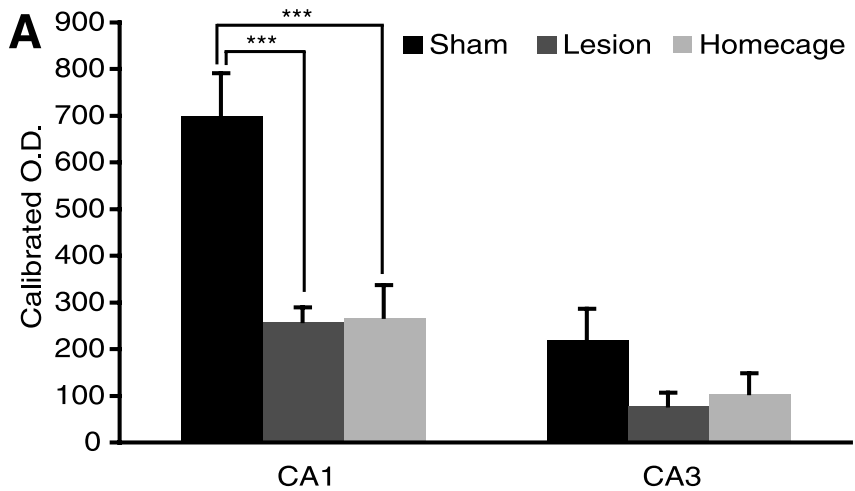

B

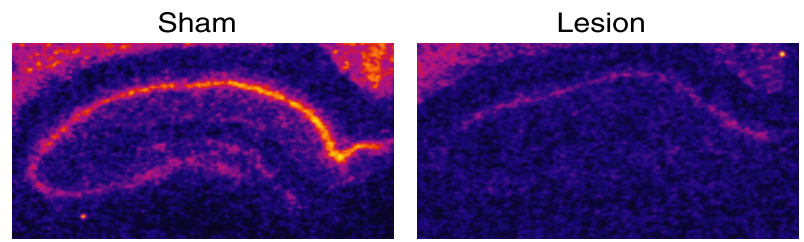

Figure 4. Fornix lesions prevent induction of hippocampal ArcmRNA after water maze training. $\boldsymbol{A}$, Sham Arc mRNA levels were significantly higher than lesion or home-cage control groups. Arc mRNA levels did not differ between lesion and home-cage control groups. $\boldsymbol{B}$, Pseudocolored autoradiographs demonstrate hippocampal Arc mRNA expression in sham and lesion animals. ${ }^{* *} p=0.001$, statistically significant. Error bars indicate SEM.

stimulation potentiated the PS amplitude equivalently in sham and lesioned animals $\left(F_{(1,6)}=0.171 ; p=0.6933\right)$ (Fig. $\left.5 A\right)$. In contrast to the effects on behavioral induction, fornix lesions did not alter Arc induction as a result of MPP stimulation (Fig. 5C). Arc mRNA levels in the molecular and granular cell layers were quantified using in situ autoradiography. Because differences in Arc expression between the granular cell layers of the upper and lower blades of the dentate gyrus were reported previously (Chawla et al., 2005), these regions were assessed separately (Fig. $5 B)$. Arc induction was equivalent in sham and fornix animals (main effect of lesion: $F_{(1,7)}=0.146, p=0.7141$; interaction effect of lesion by layer: $\left.F_{(3,21)}=0.617, p=0.6117\right)$. In both groups, Arc expression differed substantially across regions (main effect of region: $F_{(3,21)}=148.534, p<0.0001$ ) and post hoc analysis revealed a significant difference between the molecular layers of the upper and lower blades of the dentate gyrus (Scheffe's test; $p=0.0135$ ) but not the granular cell layers (Scheffe's test; $p=0.91)$ of the ipsilateral side. These data document the sensitivity of the quantitative technique used here and indicate that the induction mechanisms and transcriptional machinery remain intact in the dentate gyrus of animals with fornix lesions.

\section{Discussion}

Fornix lesions, which prevent hippocampus-dependent spatial but not hippocampus-independent cued learning, eliminate the behavioral induction of Arc transcription in the rat hippocampus. In contrast, the induction of Arc mRNA transcription by MPP potentiation is unaffected by fornix lesions. Together with the evidence that location-specific firing of hippocampal neurons is substantially preserved after fornix transection (Miller and Best, 1980; Shapiro et al., 1989), these results indicate that hippocampal firing is not sufficient to induce Arc transcription and that engaging signaling pathways specifically implicated in learning-related plasticity is required. Thus, behavior and concomitant place-field activity, on the one hand, may be decoupled
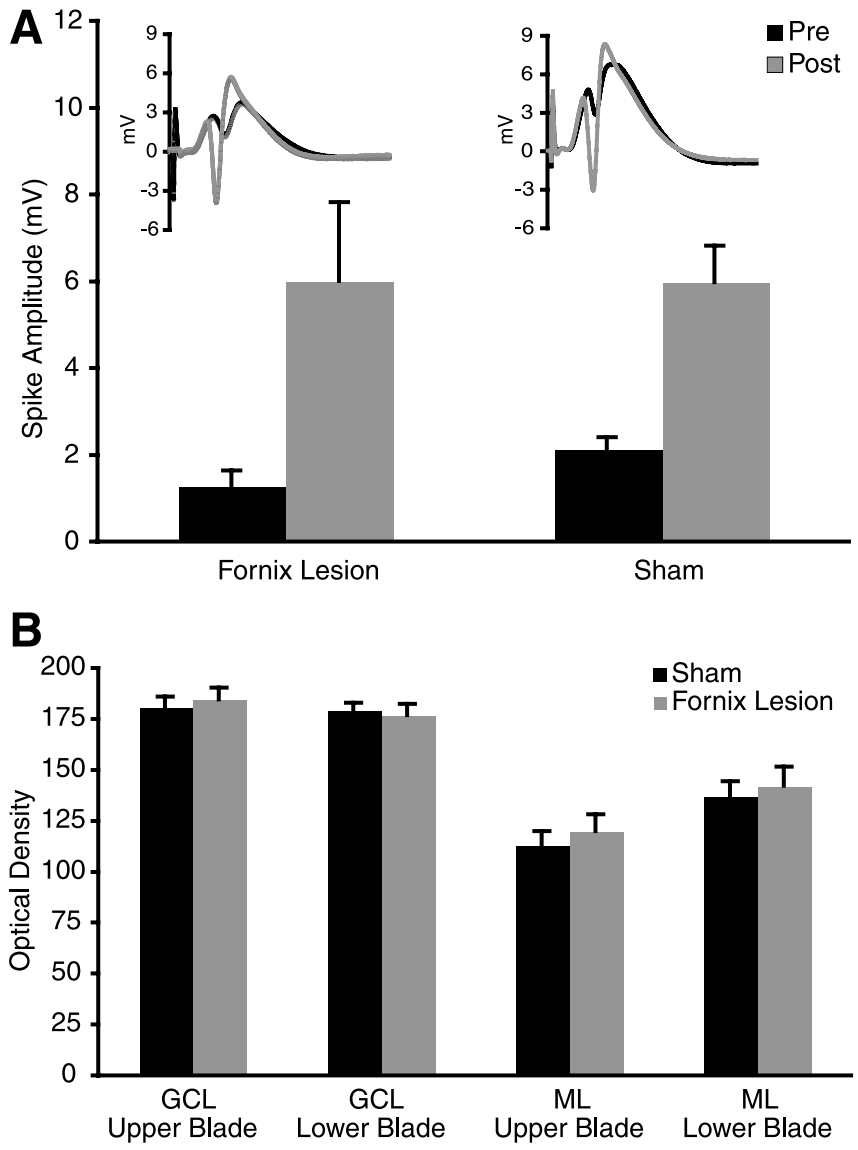

\section{C}
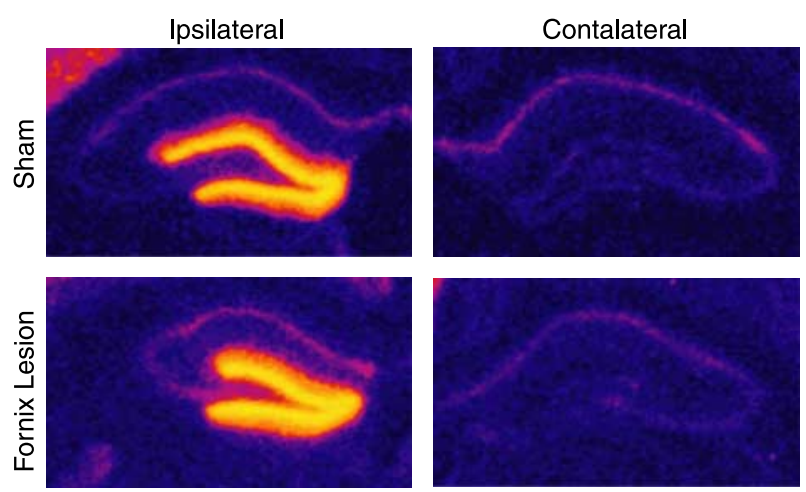

Figure 5. Fornix lesions do not prevent induction of Arc expression in response to MPP potentiation. $\boldsymbol{A}$, HFS of the MPP resulted in similar potentiation in sham and lesion animals. Representative pre- and post-HFS EPSPs from sham and lesion animals demonstrate potentiation of responses (inset). $\boldsymbol{B}$, Arc mRNA levels did not differ between sham and lesion groups. GCL, Granular cell layer; ML, molecular layer. C, Pseudocolored autoradiographs demonstrate Arc mRNA expression in the stimulated ipsilateral but not the unstimulated contralateral hippocampus of sham and lesion animals.

from plasticity, hippocampus-dependent learning, and Arc expression on the other hand.

The patterns of Arc induction across hippocampal neurons of intact animals after environmental exploration has been likened to the activation of hippocampal "place cells" recorded electrophysiologically (Guzowski et al., 1999; Vazdarjanova and Guzowski, 2004). Hippocampal neurons with place fields have also been recorded in animals learning spatial tasks in the water maze (Hollup et al., 2001a,b). Place-field activity during water and dry land tasks were similar in most respects, except for a 
significantly higher average firing rate in the water (Hollup et al., 2001b). Location-specific firing of hippocampal neurons is maintained in rats with fornix lesions (Miller and Best, 1980; Shapiro et al., 1989). Therefore, if place-field activity per se were sufficient to induce Arc, then rats with fornix lesions should show Arc expression after environmental exploration and water maze training, but they do not. The divergent effects of fornix lesions on Arc expression and place fields cannot be attributed to a global change in cellular physiology, because spike waveforms were unchanged (Shapiro et al., 1989). Nor did the lesions reduce the mean firing rate of complex spike cells, which were reported as either unchanged (Shapiro et al., 1989) or increased (Miller and Best, 1980). Although the defining properties of place fields appeared normal, the fields were more diffuse and more closely associated with proximal than distal cues in rats with fornix lesions (Miller and Best, 1980; Shapiro et al., 1989). These alterations suggest a deficit in the stable integration of environmental information after fornix lesions.

Profound behavioral deficits follow lesions of the fornix in a variety of tasks including spatial learning and memory in the water maze (Nilsson et al., 1987; Sutherland and Rodriguez, 1989), temporal memory discriminations (Olton et al., 1987), and olfactory discrimination learning (Dusek and Eichenbaum, 1997). Performance on hippocampal-independent tasks such as cue-approach learning remains intact after fornix lesions (Packard et al., 1989; McDonald and White, 1993; Sziklas and Petrides, 2002; Gaffan et al., 2003). The deficits reported here in spatial but not cue-approach learning after fornix lesions are consistent with this previous work. Additionally, the design of the behavioral task took advantage of multiple memory systems by providing parallel strategies (spatial and nonspatial) for solving the same task. All animals learned to escape efficiently to the visible platform. However, animals with fornix lesions used a cue-approach strategy, whereas intact animals used a spatial strategy.

Normal cue-approach learning by rats with fornix lesions demonstrated that motivation, perception, sensory motor integration, and motor function remained intact even as spatial learning was impaired. Furthermore, rats with fornix lesions had intact cortical connections to the hippocampus, and these inputs induced normal synaptic responses, synaptic potentiation, and Arc induction when synchronous inputs were evoked electrically. These results suggest that the cortical input that accompanies behavior is not sufficient to induce Arc in the hippocampus, but rather the combination of cortical and subcortical inputs is required to engage the hippocampal plasticity mechanisms necessary for spatial learning and Arc induction. These results also provide important clues as to how fornix lesions impair hippocampal function. Rather than suppressing neuronal activity, blocking synaptic plasticity, or preventing the expression of place fields, fornix lesions may decouple incoming patterns of cortical activity from the cellular plasticity mechanisms that it normally engages.

One possible mechanism by which fornix lesions could decouple cortical activity from learning-related plasticity mechanisms and Arc expression is by obliterating hippocampal theta rhythm. Fornix lesions destroy many neurochemical inputs to the hippocampus that are required for theta rhythm, a $4-12 \mathrm{~Hz}$ EEG oscillation that normally accompanies appetitive behavior (Rawlins et al., 1979). Theta oscillations reflect the intrinsic excitability of hippocampal neurons and have important influence on synaptic plasticity in the hippocampus (Buzsaki, 2002). Thus, bursts of electrical stimulation to the perforant path or the Schaeffer collaterals that mimic the theta rhythm (theta burst stimulation) provide an especially powerful way to induce LTP (Larson et al., 1986; Greenstein et al., 1988). Individual stimuli that arrive on the peak of the theta rhythm are more likely to produce LTP than stimuli that arrive on the trough of theta rhythm, which may produce long-term depression (Huerta and Lisman, 1996; Holscher et al., 1997; Hyman et al., 2003). According to one theory, patterns of cortical inputs are stored when they arrive on the peak of theta rhythm and are used for memory retrieval when they arrive on the trough (Hasselmo et al., 2002). The loss of theta rhythm produced by fornix lesions likely causes a temporal disorganization of hippocampal activity that should make less likely the occurrence of well-timed coincident presynaptic and postsynaptic depolarization that is required to induce LTP. For these reasons, fornix lesions could prevent cortical activity from engaging hippocampal plasticity mechanisms, in turn preventing normal information processing and Arc expression. If the loss of theta rhythmicity is the mechanism by which fornix lesions disrupt Arc expression, then pharmacological and other treatments that block theta should also prevent the induction of hippocampal Arc transcription by behavior but not potentiation.

The induction of Arc transcription has been implicated in the support of neuronal plasticity, specifically learning-related plasticity. Previous work has demonstrated that manipulations known to induce neuronal plasticity, such as HFS in vivo (Steward et al., 1998), novel environmental exploration (Guzowski et al., 1999; Vazdarjanova and Guzowski, 2004), spatial learning (Guzowski et al., 2001), and nonspatial learning (Kelly and Deadwyler, 2002, 2003) induce Arc transcription. Arc is targeted to recently activated synapses in an NMDA receptor-dependent manner in vivo (Steward and Worley, 2001), and intrahippocampal injections of Arc antisense oligonucleotides impair maintenance of LTP and memory consolidation while sparing shortterm memory (Guzowski et al., 2000). Together, these lines of evidence suggest that Arc supports the persistence of plasticityinduced synaptic alterations and contributes to long-term memory.

A specific role of Arc in supporting synaptic changes that allow learning is also suggested by studies in which Arc expression is compared across different learning paradigms. Higher levels of Arc expression in newly trained animals compared with overtrained or untrained animals suggest that Arc is involved in the acquisition rather than the expression of a learned behavior (Guzowski et al., 2001; Kelly and Deadwyler, 2002, 2003). Learning-related increases in Arc expression are differentially affected across brain structures according to task demands, so that performance on a spatial but not a cued task is correlated to hippocampal Arc mRNA levels (Guzowski et al., 2001). These studies show that Arc expression is increased in situations when new learning occurs and that Arc expression within a structure is related to the specific cognitive demands of a task.

Previous evidence, however, has not distinguished between the influences of neuronal firing that accompany learning and the plasticity that learning requires. The present study shows that when hippocampal-dependent learning and plasticity are compromised, behaviorally induced Arc expression in the hippocampus is correspondingly low. Although behavior that engages hippocampal-dependent learning in normal animals is insufficient to induce Arc transcription in fornix-lesioned animals, other manipulations that result in synaptic potentiation can induce Arc transcription. Together, the results suggest that changes in Arc expression in the hippocampus are not correlated with changes in cellular activity that accompany behavior per se, or even learning supported by other brain systems, but rather are 
correlated with learning-related plasticity that requires the hippocampus. Similarly, hippocampal LTP also increased expression of Arc protein in the dentate gyrus (Moga et al., 2004), whereas low-frequency stimulation that produced either the same number of PSs or presynaptic volleys induced neither potentiation nor Arc. These data indicate that the expression of Arc protein is also linked to plasticity-inducing stimulation.

These findings further suggest that Arc mRNA expression patterns are more closely tied to learning-related plasticity than to correlates of neuronal activity. Arc may therefore be more suitable for examining learning-related plasticity patterns that occur during task acquisition than for examining patterns of activity that occur during the performance of a well learned task. Thus, Arc expression could be used in conjunction with markers of neuronal activity to contrast populations of cells that fire during behavior from those that are involved in learning-related plasticity.

Many other IEGs are induced behaviorally (c-fos, Zif268, Homer 1a, etc.). The relative contributions of neuronal activation and learning-related plasticity to the behavioral induction of these other IEGs remains undefined. The experiments described here provide a method to assess the extent to which the behaviorally induced transcription of other IEGs is linked specifically to the engagement of learning-related plasticity or more broadly with neuronal activation.

\section{References}

Abraham WC, Christie BR, Logan B, Lawlor P, Dragunow M (1994) Immediate early gene expression associated with the persistence of heterosynaptic long-term depression in the hippocampus. Proc Natl Acad Sci USA 91:10049-10053.

Alberini CM, Ghirardi M, Metz R, Kandel ER (1994) C/EBP is an immediate-early gene required for the consolidation of long-term facilitation in Aplysia. Cell 76:1099-1114.

Buzsaki G (2002) Theta oscillations in the hippocampus. Neuron 33:325-340.

Buzsaki G, Gage FH (1989) Absence of long-term potentiation in the subcortically deafferented dentate gyrus. Brain Res 484:94-101.

Cassel JC, Duconseille E, Jeltsch H, Will B (1997) The fimbria-fornix/cingular bundle pathways: a review of neurochemical and behavioural approaches using lesions and transplantation techniques. Prog Neurobiol 51:663-716.

Chawla MK, Guzowski JF, Ramirez-Amaya V, Lipa P, Hoffman KL, Marriott LK, Worley PF, McNaughton BL, Barnes CA (2005) Sparse, environmentally selective expression of Arc RNA in the upper blade of the rodent fascia dentata by brief spatial experience. Hippocampus 15:579-586.

Dusek JA, Eichenbaum H (1997) The hippocampus and memory for orderly stimulus relations. Proc Natl Acad Sci USA 94:7109-7114.

Flexner JB, Flexner LB, Stellar E (1963) Memory in mice as affected by intracerebral puromycin. Science 141:57-59.

Frey U, Krug M, Reymann KG, Matthies H (1988) Anisomycin, an inhibitor of protein synthesis, blocks late phases of LTP phenomena in the hippocampal CA1 region in vitro. Brain Res 452:57-65.

Gaffan EA, Bannerman DM, Healey AN (2003) Learning associations between places and visual cues without learning to navigate: neither fornix nor entorhinal cortex is required. Hippocampus 13:445-460.

Greenstein YJ, Pavlides C, Winson J (1988) Long-term potentiation in the dentate gyrus is preferentially induced at theta rhythm periodicity. Brain Res 438:331-334.

Guzowski JF, McNaughton BL, Barnes CA, Worley PF (1999) Environment-specific expression of the immediate-early gene Arc in hippocampal neuronal ensembles. Nat Neurosci 2:1120-1124.

Guzowski JF, Lyford GL, Stevenson GD, Houston FP, McGaugh JL, Worley PF, Barnes CA (2000) Inhibition of activity-dependent arc protein expression in the rat hippocampus impairs the maintenance of long-term potentiation and the consolidation of long-term memory. J Neurosci 20:3993-4001.

Guzowski JF, Setlow B, Wagner EK, McGaugh JL (2001) Experiencedependent gene expression in the rat hippocampus after spatial learning: a comparison of the immediate-early genes Arc, c-fos, and zif268. J Neurosci 21:5089-5098.

Hasselmo ME, Bodelon C, Wyble BP (2002) A proposed function for hippocampal theta rhythm: separate phases of encoding and retrieval enhance reversal of prior learning. Neural Comput 14:793-817.

Hedreen JC, Bacon SJ, Price DL (1985) A modified histochemical technique to visualize acetylcholinesterase-containing axons. J Histochem Cytochem 33:134-140.

Hollup SA, Molden S, Donnett JG, Moser MB, Moser EI (2001a) Accumulation of hippocampal place fields at the goal location in an annular watermaze task. J Neurosci 21:1635-1644.

Hollup SA, Molden S, Donnett JG, Moser MB, Moser EI (2001b) Place fields of rat hippocampal pyramidal cells and spatial learning in the watermaze. Eur J Neurosci 13:1197-1208.

Holscher C, Anwyl R, Rowan MJ (1997) Stimulation on the positive phase of hippocampal theta rhythm induces long-term potentiation that can be depotentiated by stimulation on the negative phase in area CA1 in vivo. J Neurosci 17:6470-6477.

Huerta PT, Lisman JE (1996) Low-frequency stimulation at the troughs of theta-oscillation induces long-term depression of previously potentiated CA1 synapses. J Neurophysiol 75:877-884.

Hyman JM, Wyble BP, Goyal V, Rossi CA, Hasselmo ME (2003) Stimulation in hippocampal region CA1 in behaving rats yields long-term potentiation when delivered to the peak of theta and long-term depression when delivered to the trough. J Neurosci 23:11725-11731.

Kelly MP, Deadwyler SA (2002) Acquisition of a novel behavior induces higher levels of Arc mRNA than does overtrained performance. Neuroscience 110:617-626.

Kelly MP, Deadwyler SA (2003) Experience-dependent regulation of the immediate-early gene arc differs across brain regions. J Neurosci 23:6443-6451.

Larson J, Wong D, Lynch G (1986) Patterned stimulation at the theta frequency is optimal for the induction of hippocampal long-term potentiation. Brain Res 368:347-350.

Lyford GL, Yamagata K, Kaufmann WE, Barnes CA, Sanders LK, Copeland NG, Gilbert DJ, Jenkins NA, Lanahan AA, Worley PF (1995) Arc, a growth factor and activity-regulated gene, encodes a novel cytoskeletonassociated protein that is enriched in neuronal dendrites. Neuron 14:433-445.

McDonald RJ, White NM (1993) A triple dissociation of memory systems: hippocampus, amygdala, and dorsal striatum. Behav Neurosci 107:3-22.

Miller VM, Best PJ (1980) Spatial correlates of hippocampal unit activity are altered by lesions of the fornix and endorhinal cortex. Brain Res 194:311-323.

Moga DE, Calhoun ME, Chowdhury A, Worley P, Morrison JH, Shapiro ML (2004) Activity-regulated cytoskeletal-associated protein is localized to recently activated excitatory synapses. Neuroscience 125:7-11.

Montarolo PG, Goelet P, Castellucci VF, Morgan J, Kandel ER, Schacher S (1986) A critical period for macromolecular synthesis in long-term heterosynaptic facilitation in Aplysia. Science 234:1249-1254.

Morrow BA, Elsworth JD, Inglis FM, Roth RH (1999) An antisense oligonucleotide reverses the footshock-induced expression of fos in the rat medial prefrontal cortex and the subsequent expression of conditioned fear-induced immobility. J Neurosci 19:5666-5673.

Nakao K, Ikegaya Y, Yamada MK, Nishiyama N, Matsuki N (2001) Spatial performance correlates with long-term potentiation of the dentate gyrus but not of the CA1 region in rats with fimbria-fornix lesions. Neurosci Lett 307:159-162.

Nilsson OG, Shapiro ML, Gage FH, Olton DS, Bjorklund A (1987) Spatial learning and memory following fimbria-fornix transection and grafting of fetal septal neurons to the hippocampus. Exp Brain Res 67:195-215.

Olton DS, Meck WH, Church RM (1987) Separation of hippocampal and amygdaloid involvement in temporal memory dysfunctions. Brain Res 404:180-188.

Packard MG, Hirsh R, White NM (1989) Differential effects of fornix and caudate nucleus lesions on two radial maze tasks: evidence for multiple memory systems. J Neurosci 9:1465-1472.

Paxinos G, Watson C (1986) The rat brain in stereotaxic coordinates, Ed 2. Sydney: Academic.

Pinaud R, Penner MR, Robertson HA, Currie RW (2001) Upregulation of the immediate early gene arc in the brains of rats exposed to environmen- 
tal enrichment: implications for molecular plasticity. Brain Res Mol Brain Res 91:50-56.

Rawlins JN, Feldon J, Gray JA (1979) Septo-hippocampal connections and the hippocampal theta rhythm. Exp Brain Res 37:49-63.

Shapiro ML, Simon DK, Olton DS, Gage III FH, Nilsson O, Bjorklund A (1989) Intrahippocampal grafts of fetal basal forebrain tissue alter place fields in the hippocampus of rats with fimbria-fornix lesions. Neuroscience 32:1-18.

Steward O, Worley PF (2001) Selective targeting of newly synthesized Arc mRNA to active synapses requires NMDA receptor activation. Neuron 30:227-240.

Steward O, Wallace CS, Lyford GL, Worley PF (1998) Synaptic activation causes the mRNA for the IEG Arc to localize selectively near activated postsynaptic sites on dendrites. Neuron 21:741-751.

Sutherland RJ, Rodriguez AJ (1989) The role of the fornix/fimbria and some related subcortical structures in place learning and memory. Behav Brain Res 32:265-277.

Swanson LW, Cowan WM (1977) An autoradiographic study of the organization of the efferent connections of the hippocampal formation in the rat. J Comp Neurol 172:49-84.

Sziklas V, Petrides M (2002) Effects of lesions to the hippocampus or the fornix on allocentric conditional associative learning in rats. Hippocampus 12:543-550.

Temple MD, Worley PF, Steward O (2003) Visualizing changes in circuit activity resulting from denervation and reinnervation using immediate early gene expression. J Neurosci 23:2779-2788.

Vazdarjanova A, Guzowski JF (2004) Differences in hippocampal neuronal population responses to modifications of an environmental context: evidence for distinct, yet complementary, functions of CA3 and CA1 ensembles. J Neurosci 24:6489-6496. 